\title{
Frequency of takeaway food consumption and its association with major food group consumption, anthropometric measures and blood pressure during adolescence
}

\author{
Bamini Gopinath $^{1 *}$, Victoria M. Flood ${ }^{2}$, George Burlutsky ${ }^{1}$, Jimmy C. Y. Louie ${ }^{3}$, Louise A. Baur ${ }^{4,5}$ \\ and Paul Mitchell ${ }^{1}$ \\ ${ }^{1}$ Centre for Vision Research, Department of Ophthalmology and The Westmead Institute, University of Sydney, Australia \\ ${ }^{2}$ Faculty of Health Sciences, University of Sydney, St Vincent's Hospital, Sydney, NSW 2010, Australia \\ ${ }^{3}$ School of Molecular Bioscience, Faculty of Sciences, University of Sydney, Sydney, NSW 2006, Australia \\ ${ }^{4}$ University of Sydney Clinical School, The Children's Hospital at Westmead, Sydney, NSW 2145, Australia \\ ${ }^{5}$ School of Public Health, University of Sydney, Sydney, NSW 2006, Australia
}

(Submitted 20 August 2015 - Final revision received 14 February 2016 - Accepted 17 February 2016 - First published online 5 April 2016)

\section{Abstract}

We prospectively assessed the (1) frequency and socio-economic correlates of takeaway food consumption during adolescence; and (2) association between frequent takeaway food consumption with intakes of major food groups and anthropometric measures and blood pressure (BP). In total, 699 Sydney schoolchildren (380 girls and 319 boys) who had dietary data at both 12 and 17 years of age were included for analyses. Takeaway food consumption was self-reported and based on a single question. Anthropometric measures and BP were collected. The proportion of participants who ate takeaway foods once per week or more increased significantly over 5 years from the age of 12 to 17 years: $35 \cdot 5-44 \cdot 1 \%(P<0 \cdot 0001)$. In total, 12 -year-old girls compared with boys had reduced odds of takeaway foods once per week or more at the age of 17 years $(P=0.01)$, multivariable-adjusted OR 0.63 (95\% CI 0.44, 0.90). In total, 12-year-old children who ate takeaway foods once per week or more had significantly lower mean fruit $(220.3 v .253 .0 \mathrm{~g} / \mathrm{d} ; P=0.03)$ and vegetable consumption $(213.2 v .247 .7 \mathrm{~g} / \mathrm{d}$; $P=0.004$ ), 5 years later (at 17 years of age). Frequent takeaway food consumption at the age of 12 years was not associated with anthropometric indices and $\mathrm{BP}$ at the age of 17 years. Consumption of takeaway foods became more frequent during adolescence, particularly among boys, and it was associated with reduced intake of fruits and vegetables.

\section{Key words: Takeaway foods: Socio-economic staus: Children: Adolescence: Fruits: Vegetables: Cohorts}

Consumption of takeaway or fast food by children and adolescents has become more prevalent around the world ${ }^{(1,2)}$. This may be because of the relatively cheap, accessible fast food, food marketing and changed family lifestyles, with both parents working ${ }^{(3)}$. Takeaway foods are often high in SFA, are energy dense and have low micronutrient content ${ }^{(4-6)}$. Moreover, a high frequency of takeaway and fast food consumption is linked to poorer diet quality, including a lower intake of vegetables $^{(3,4,7,8)}$, wholegrains ${ }^{(9)}$, low-fat dairy products ${ }^{(9)}$ and fruit ${ }^{(3,4,7)}$, and a higher intake of total fat and $\mathrm{SFA}^{(8)}, \mathrm{Na}^{(8)}$ and sugar-sweetened carbonated soft drinks ${ }^{(3,7,8)}$. Higher intake of fats and Na could modulate increased blood pressure (BP) and BMI. Indeed, a recent study of young adults showed that those with prehypertension or hypertension tended to eat more meals away from home per week and have higher $\mathrm{BMI}^{(8,10)}$.

There is a growing body of literature to suggest that socioeconomic status strongly influences exposure to takeaway foods ${ }^{(4)}$. Specifically, in developed countries, takeaway food outlets are found more often in areas of lower socio-economic status $^{(4)}$. One of the hypothesised reasons is that those of lower socio-economic status eat more fast foods because of the relative cheapness of these foods ${ }^{(4,11)}$. However, most studies examining the links between socio-economic status and takeaway food consumption in children and adolescents have been undertaken in the USA and $\mathrm{UK}^{(4)}$. Similarly, most published literature reporting on the associations between frequent takeaway food consumption and adiposity, and the intake of other food groups, has come from childhood studies conducted in these two countries and are largely cross-sectional. Findings from these studies might not be generalisable to other countries, including Australia. Therefore, the key aims of this cohort study of Australian schoolchildren aged 12 and 17 years were to prospectively examine the following: (1) pattern and change in takeaway food consumption during adolescence; (2) socio-demographic factors

Abbreviation: BP, blood pressure.

* Corresponding author: B. Gopinath, fax +61 2 98458345, email bamini.gopinath@sydney.edu.au 
associated with takeaway food consumption; (3) independent associations between frequency of takeaway food consumption and intakes of fruits, vegetables and dairy foods, as well as the consumption of soft drinks; and (4) associations of takeaway food consumption with adiposity indices (BMI, waist circumference, and percentage of body fat), weight status and BP.

\section{Methods}

\section{Study population}

The Sydney Childhood Eye Study is a population-based survey of eye conditions and other health outcomes in schoolchildren living within the Sydney metropolitan area, Australia. It was approved by the Human Research Ethics Committee, University of Sydney, the Department of Education and Training, and the Catholic Education Office, NSW, Australia ${ }^{(12)}$. We obtained informed written consent from at least one parent of each child, as well as verbal assent and written consent from each child/adolescent before the examinations. Study methods have been previously described ${ }^{(12)}$. Briefly, students with a mean age 12.7 years in a stratified random cluster sample of twenty-one high schools across Sydney were eligible to participate. Stratification was based on socio-economic status data and led to a proportional mix of public, private or religious high schools. Of the 3144 eligible 12-year-old children, 2367 were given parental permission to participate and 2353 underwent examinations $(74.9 \%)$. Data for the 12-year-old cohort were collected during 2004-2005 and then 5 years later during 2009-2011; 1216 were re-examined ( $51.7 \%$ of baseline participants).

\section{Dietary data assessment}

Dietary data were collected using a 120-item self-administered, semi-quantitative FFQ, designed for specific use in Australian children and adolescents ${ }^{(13)}$, administered at ages 12 and 17 years. An allowance for seasonal variation of fruit and vegetables was made during analysis by weighting seasonal fruits and vegetables. The validity of this FFQ has previously been reported ${ }^{(13)}$. FFQ items were translated into daily food and nutrient intakes using a purpose-built query in Microsoft Access 2007 , using various nutrient databases ${ }^{(14-16)}$.

Data on the frequency of takeaway food consumption were also obtained from the FFQ administered at ages 12 and 17 years. At both baseline and 5-year follow-up, study participants were asked the following question: 'How often do you eat takeaway foods (e.g. Chinese, fish and chips, hamburger, and chips/fries, pizza)?' Response options were as follows: 'never', 'less than once per week', '1-2/week', '3-4/week', '5-6/week' or ' 1 or more per $d$ '. Information is not available on the reliability or validity of these questions, but similar frequencybased questions have been used in other studies ${ }^{(11,17)}$. Responses in the two least frequent categories of the above question, never ( $n$ 23) and less than once per week ( $n$ 429), were collapsed for analysis and formed one response variable, that is, 'less than once per week'. As only about 5-6\% consumed takeaway foods more than 1-2 times per week, responses in the three most frequent categories, $1-2 /$ week ( $n$ 230), 3-4/week ( $n$ 14), 5-6/week ( $n$ 2) and $\geq 1 / \mathrm{d}(n 1)$, were also combined for analysis and is termed 'once per week or more'. Hence, this led to dichotomous outcome variables that were subsequently used in all analyses, that is, less than once per week and once per week or more.

We examined the validity of the summation of takeaway-type foods reported in the long FFQ (120 items) compared with the short takeaway food questions ${ }^{(2)}$. The foods included in the created takeaway item from the long FFQ were as follows: hamburger, pizza, pies or sausage rolls, hot dogs, savoury pastries, fried potatoes such as hash browns and hot chips. Of these items only the hot chips item was specifically identified as purchased away from home and not home prepared. Comparison of the created takeaway food frequency from the long FFQ reported a fair Spearman's correlation coefficient of 0.340 for the 12 year old data set and 0.429 for the 17 -year-old data set (both with $P<0 \cdot 0001$ ). However, the long FFQ tended to provide a higher estimate of takeaway food use: when the data were categorised into those consuming $<1$ /week to those consuming $\geq 1$ times/week, as used in the manuscript, only $27 \%$ of the short data item from the 12-year-old data set had takeaway foods less than once per week, and $40 \%$ of the 17-year-old data set had takeaway foods less than once per week. By contrast, those who reported consuming $\geq 1$ times/week in the short question were also likely to have this result in the long FFQ: $91 \%$ in the 12-year-old data set and $87 \%$ in the 17 -year-old data set.

\section{Assessment of adiposity and blood pressure}

Anthropometric measures and BP were collected at both baseline and 5-year follow-up. Each participant's weight and body fat percentage were measured using a Body Composition Analyser (model TBF-300; Tanita). Height was measured to the nearest $0.1 \mathrm{~cm}$ with shoes off using a freestanding SECA height rod (model 220; Hamburg). Weight in kilograms was measured to the nearest $0 \cdot 1 \mathrm{~kg}$ using a standard portable weighing machine (Wedderburn Scales), after removing any heavy clothing. Anthropometric measures were recorded up to 2 decimal points. Overweight and obesity categories were defined using the International Obesity Taskforce age- and sexspecific cut-off points ${ }^{(18)}$. BP was measured on the school premises according to a standard protocol ${ }^{(12)}$. After 5-min resting, BP was measured in a seated position using an automated sphygmomanometer (HEM 907; Omron Healthcare Inc.) with appropriate cuff size. We followed general recommendations on selecting cuff size to ensure that the bladder length was approximately $80 \%$ and width was at least $40 \%$ of the arm circumference, covering the upper arm without obscuring the antecubital fossa ${ }^{(19)}$. Three separate BP measurements were taken (with a resting time of 5 min between each measurement) and averaged for analysis ${ }^{(20)}$. Mean arterial BP was calculated as one-third of the systolic plus two-thirds of the diastolic BP.

\section{Assessment of potential confounders}

Information on all covariates was obtained both at baseline (age 12 years) and at the 5-year follow-up (age 17 years). Parents also completed a comprehensive 193-item questionnaire. 
Socio-demographic information covering ethnicity of the child, country of birth, education, occupation and parental age of both parents was collected. The ethnicity of the child was determined only if both parents shared that ethnic origin. Otherwise, children were placed in a mixed ethnicity category. Ethnicity was classified on the basis of self-identification by the parents, combined with information about the place of birth of the child ${ }^{(21)}$. The questions relating to physical activity comprised a list of nine common activities in which Sydney school-aged children typically participated such as athletics, swimming, soccer and so on. ${ }^{(22)}$. Children self-reported the usual number of hours per week they spent in each of these activities and whether the activity was done outdoors or indoors (hall gym, classroom). The time spent in each activity was summated, and the average hours per $\mathrm{d}$ spent in total physical activity was calculated. Total screen time $(\mathrm{h} / \mathrm{d})$ was calculated as the time reported that was spent on the following activities: watching TV, playing video games and using a computer for both recreational and educational purposes ${ }^{(22)}$

\section{Statistical analyses}

Statistical analyses were performed using SAS (version 9.2; SAS Institute). Descriptive statistics were used to report the proportion of adolescents consuming takeaway foods. Paired sample $t$ tests were used to assess changes in individual intakes over time. We used mixed models to adjust for cluster sampling effects. Logistic regression analysis was used to assess longitudinal associations between various socio-economic correlates (age, sex, ethnicity, parental education and employment status) at the age of 12 years and consuming takeaway foods once or more per week at the age of 17 years. All socio-economic correlates were included in one model. ANCOVA was used to calculate differences in mean fruit, vegetable and total dairy food consumption, as well as mean anthropometric measures and BP, adjusting for the relevant significant covariates. The association between takeaway food consumption and frequency of soft drink intake was assessed using logistic regression modelling. Only those potential confounders that significantly modified the effect of takeaway food consumption in age- and sex-adjusted models, or significantly predicted the outcome variable (i.e. either major food group consumption, adiposity or BP measures), were included in subsequent multivariable analyses.

\section{Results}

Of the 2353 12-year-old children who were examined at baseline, 2078 had information on takeaway meal consumption (i.e. had completed the FFQ). At the 5-year follow-up, of the 1216 who were examined at the age of 17 years, 993 had answered the question on takeaway meal consumption. Of those surveyed at both baseline and 5-year follow-up, 699 participants had complete information on consumption of takeaway meals at both time points and, hence, were included in prospective analyses - that is, assessing the temporal change in takeaway meal consumption during adolescence. We compared the baseline characteristics (at age 12 years) of those who completed the FFQ (i.e. had information on frequency of takeaway foods) and those who did not complete the FFQ at the age of 17 years. This analysis found that those who had complete information on takeaway food consumption at the age of 17 years compared with those who had missing FFQ data at the age of 17 years were more likely to be female $(54.4 \mathrm{v}$. $43.4 \% ; P=0.001)$; Caucasian $(65.0 \% ; P=0.0002)$; have higher percentage of body fat (20.7 v. 18.9\%; $P=0.002)$; and higher systolic BP (113.2 v. $111.4 \mathrm{mmHg} ; P=0 \cdot 01)$. No other significant differences were observed in baseline characteristics between these two groups (data not shown). Overall, the proportion of study participants who ate takeaway meals once or more per week increased significantly over the 5 years from 35.5 to $44.1 \%(P<0 \cdot 0001 ;$ Table 1$)$. This appeared to be primarily driven by a significant increase in the proportion of boys who ate takeaway meals once or more/week, between the ages of 12 and 17 years $(13.8 \%$ increase; $P<0.0001)$. A non-significant increase in the proportion of girls eating takeaway meals once or more per week was observed during the 5-year follow-up $(P=0 \cdot 05$; Table 1$)$. Multivariable regression analyses showed that 12 -year-old girls compared with boys had $37 \%$ reduced odds of takeaway foods once per week or more at the age of 17 years $(P=0 \cdot 01$; Table 2$)$. No other baseline socio-economic correlates independently predicted frequency of takeaway food consumption at the 5-year follow-up (Table 2).

Unadjusted analysis showed that participants who ate takeaway foods once per week or more compared with less than once a week had significantly lower fruit $(204.1 v .247 .4 \mathrm{~g} / \mathrm{d}$; $P=0.002)$ and vegetable consumption $(222.7 v .256 .5 \mathrm{~g} / \mathrm{d}$; $P=0.01)$ at the age of 17 years. Multivariable analysis also showed that 12 -year-old children who were frequent consumers of takeaway foods compared with those who ate takeaway foods infrequently had significantly lower mean fruit $(220.3$ v. $253.0 \mathrm{~g} / \mathrm{d} ; \quad P=0.03)$ and vegetable consumption (213.2 v. $247.7 \mathrm{~g} / \mathrm{d} ; P=0.004), 5$ years later (Table 3 ). Additional analysis showed that 12 -year-old children who ate takeaway foods once per week or more had $7 \cdot 5$-fold increased odds of eating takeaway foods once per week or more 5 years later at the age of 17 years, multivariable-adjusted OR 7.52 (95\% CI $3 \cdot 64,15 \cdot 54)$. Temporal analysis showed that frequent consumption of takeaway meals was not significantly associated with BP (Table 4) or anthropometric measures (Table 5) at the

Table 1. Consumption of takeaway meals at 12 and 17 years of age among participants of the Sydney Childhood Eye Study during 2004-2005 to 2009-2011

(Numbers and percentages)

\begin{tabular}{|c|c|c|c|c|c|}
\hline \multirow[b]{2}{*}{ Consumption } & \multicolumn{2}{|c|}{12 years } & \multicolumn{2}{|c|}{17 years } & \multirow[b]{2}{*}{$P$} \\
\hline & $n$ & $\%$ & $n$ & $\%$ & \\
\hline \multicolumn{6}{|l|}{ All $(n$ 699) } \\
\hline Less than once per week & 452 & $64 \cdot 7$ & 390 & 55.8 & $<0.0001$ \\
\hline Once per week or more & 247 & $35 \cdot 3$ & 309 & $44 \cdot 2$ & \\
\hline \multicolumn{6}{|l|}{ Girls $(n 380)$} \\
\hline Less than once per week & 249 & $65 \cdot 5$ & 231 & $60 \cdot 8$ & 0.05 \\
\hline Once per week or more & 131 & 34.5 & 149 & 39.2 & \\
\hline \multicolumn{6}{|l|}{ Boys ( $n$ 319) } \\
\hline Less than once per week & 203 & $63 \cdot 6$ & 159 & 49.8 & $<0.0001$ \\
\hline Once per week or more & 116 & $36 \cdot 4$ & 160 & $50 \cdot 2$ & \\
\hline
\end{tabular}


age of 17 years. Further, 12-year-old children who ate takeaway foods once per week or more compared with those who ate takeaway foods infrequently did not have significantly higher odds of being overweight or obese at the age of 17 years, multivariable-adjusted OR 0.99 (95\% CI 0.59 , 1.66) and OR 1.59 (95\% CI 0.86, 2.94), respectively.

\section{Discussion}

This epidemiological study showed that Australian schoolchildren appreciably increased their consumption of takeaway

Table 2. Multivariable logistic regression model showing the temporal association between socio-economic correlates at 12 years of age and eating takeaway meals once or more per week ${ }^{\star}$ at the age of 17 years among participants of the Sydney Childhood Eye Study (Odds ratios and $95 \%$ confidence intervals; $n$ 699)

\begin{tabular}{|c|c|c|}
\hline \multirow[b]{2}{*}{ Variables } & \multicolumn{2}{|c|}{$\begin{array}{c}\text { Eating takeaway meals once or } \\
\text { more per week at the age of } \\
17 \text { years }(n 247)\end{array}$} \\
\hline & OR & $95 \% \mathrm{Cl}$ \\
\hline$\underset{P}{\text { Age (years) (per year) }}$ & \multicolumn{2}{|c|}{0.17} \\
\hline Sex & & \\
\hline $\begin{array}{l}\text { Male }(n \text { 319) } \\
\text { Female }(n 380) \\
P\end{array}$ & $\begin{array}{l}1.0 \\
0.63\end{array}$ & $\begin{array}{c}\text { Ref. } \\
0.44,0.90\end{array}$ \\
\hline Ethnicity (\%) & & \\
\hline $\begin{array}{l}\text { Caucasian ( } n \text { 454) } \\
\text { East Asian }(n 113) \\
P\end{array}$ & $\begin{array}{l}1.0 \\
1.13\end{array}$ & $\begin{array}{l}\text { Ref. } \\
0.67,1.92\end{array}$ \\
\hline $\begin{array}{l}\text { South Asian }(n 36) \\
P\end{array}$ & \multicolumn{2}{|c|}{$0.74^{0.46,1.73}$} \\
\hline $\begin{array}{l}\text { Middle Eastern }(n 36) \\
\quad P\end{array}$ & \multicolumn{2}{|c|}{0.67} \\
\hline $\begin{array}{l}\text { Others/mixed }(n 60) \\
\quad P\end{array}$ & \multicolumn{2}{|c|}{0.61} \\
\hline Parental education & & \\
\hline $\begin{array}{l}\text { University ( } n \text { 329) } \\
\text { Less than university ( } n \text { 370) }\end{array}$ & $\begin{array}{l}1.0 \\
1.20\end{array}$ & $\begin{array}{c}\text { Ref. } \\
0 \cdot 79,1 \cdot 81\end{array}$ \\
\hline \multicolumn{3}{|l|}{ Parental employment status } \\
\hline $\begin{array}{l}\text { Full-time employment }(n 598) \\
\text { Not in full-time employment }(n \text { 101) } \\
P\end{array}$ & $\begin{array}{l}1.0 \\
0.83\end{array}$ & $\begin{array}{c}\text { Ref. } \\
0.39,1.76\end{array}$ \\
\hline
\end{tabular}

Ref., referent value.

* In comparison to the reference group, who were participants who consumed takeaway meals less than once per week at age 17 years $(n 452)$. foods (e.g. hamburgers, chips/fries, pizza) during adolescence. Girls compared with boys were less likely to eat takeaway foods once per week or more during adolescence. Lower mean vegetable and fruit consumption was observed among 17-yearold schoolchildren who ate takeaway foods once per week or more at the age of 12 years. Eating takeaway foods at least weekly was not significantly associated with BP measures or with adiposity during the 5-year follow-up.

Around a third of 12-year-old children consumed takeaway foods at least weekly, whereas just over two-fifths of 17-yearold children ate takeaway foods once or more per week. An international study conducted in 2002-2003 also found that about 39\% of children aged 13-14 years reported frequent consumption of takeaway foods ${ }^{(23)}$. However, our proportions are slightly lower than those reported by another UK study conducted during $2010^{(3)}$, which showed that $50 \%$ of schoolchildren aged 11-14 years purchased foods or drinks from takeaway outlets twice or more per week. The significant increase in proportion of schoolchildren who frequently ate takeaway foods over the 5 years in our study is of concern. Changes in the mean intake of a food with age is likely to reflect the change in frequency of consumption ${ }^{(24,25)}$.

Our study findings are in agreement with previous UK studies that boys were more likely than girls to eat takeaway foods during adolescence ${ }^{(3,11)}$. Further, this observation is supported by our previous publication ${ }^{(26)}$, which showed that $19 \%$ of boys and $23 \%$ of girls engaged in more obesogenic behavioural risk factors such as high snack intake (e.g. potato crisps, chocolate) and high soft-drink consumption. However, the sex-specific

Table 4. Association between frequency of takeaway food consumption at the age of 12 years and adjusted mean blood pressure (BP; $\mathrm{mmHg}$ ) 5 years later at the age of 17 years in the Sydney Childhood Eye Study (Multivariable-adjusted mean values and 95\% confidence intervals)

\begin{tabular}{|c|c|c|c|c|}
\hline & \multicolumn{2}{|c|}{$\begin{array}{l}\text { Systolic BP } \\
\text { at } 17 \text { years }\end{array}$} & \multicolumn{2}{|c|}{$\begin{array}{l}\text { Diastolic BP } \\
\text { at } 17 \text { years }\end{array}$} \\
\hline & Mean* & $95 \% \mathrm{Cl}$ & Mean* & $95 \% \mathrm{Cl}$ \\
\hline \multicolumn{5}{|c|}{ Eating takeaway foods at the age of 12 years } \\
\hline Less than once per week ( $n$ 446) & $121 \cdot 7$ & 1.0 & $70 \cdot 3$ & 0.9 \\
\hline Once or more per week ( $n$ 242) & $122 \cdot 2$ & 1.1 & $70 \cdot 4$ & 0.9 \\
\hline$P$ & \multicolumn{2}{|c|}{0.61} & \multicolumn{2}{|c|}{0.95} \\
\hline
\end{tabular}

* Adjusted for age, sex, ethnicity, parental education, parental employment, BMI, height, physical activity and energy intake.

Table 3. Temporal association between eating takeaway foods at the age of 12 years and adjusted mean dietary intakes of fruits, vegetables and dairy foods, and soft drink consumption 5 years later at the age of 17 years

(Multivariable-adjusted mean values and $95 \%$ confidence intervals; multivariable-adjusted odds ratios and $95 \%$ confidence intervals)

\begin{tabular}{|c|c|c|c|c|c|c|c|c|c|}
\hline & \multicolumn{2}{|c|}{ Fruits $(\mathrm{g} / \mathrm{d})$} & \multicolumn{2}{|c|}{ Vegetables (g/d) } & \multicolumn{3}{|c|}{ Total dairy product intake (mean serves/d) } & \multicolumn{2}{|c|}{ Soft drinks† } \\
\hline & Mean* & $95 \% \mathrm{Cl}$ & Mean* & $95 \% \mathrm{Cl}$ & Mean* & & $95 \% \mathrm{Cl}$ & $\mathrm{OR}^{*}$ & $95 \% \mathrm{Cl}$ \\
\hline \multicolumn{10}{|c|}{ Eating takeaway meals at the age of 12 years } \\
\hline Less than once per week ( $n$ 452) & 253.0 & $224 \cdot 6,282 \cdot 5$ & 247.7 & $222 \cdot 6,272 \cdot 8$ & 1.41 & & $1 \cdot 20,1.63$ & 1.0 & Ref. \\
\hline Once or more per week ( $n$ 247) & $220 \cdot 3$ & $187 \cdot 0,253 \cdot 7$ & $213 \cdot 2$ & $185 \cdot 0,241 \cdot 4$ & 1.67 & & $1 \cdot 12,1.60$ & 0.97 & $0.64,1.47$ \\
\hline$P$ & & 0.03 & & 0.004 & & 0.63 & & & 0.88 \\
\hline
\end{tabular}

Ref., referent value.

* Adjusted for baseline age, sex, ethnicity, parental education, parental employment and physical activity.

$\dagger$ Consuming soft drinks once or more per $d$. 
Table 5. Longitudinal association between frequency of takeaway food consumption at the age of 12 years and adjusted mean BMI, waist circumference, and percentage of body fat at the age of 17 years in the Sydney Childhood Eye Study (Multivariable-adjusted mean and $95 \%$ confidence intervals)

\begin{tabular}{|c|c|c|c|c|c|c|}
\hline & \multicolumn{2}{|c|}{$\mathrm{BMI}\left(\mathrm{kg} / \mathrm{m}^{2}\right)$} & \multicolumn{2}{|c|}{ Waist circumference $(\mathrm{cm})$} & \multicolumn{2}{|c|}{ Percentage of body fat (\%) } \\
\hline & Mean* $^{*}$ & $95 \% \mathrm{Cl}$ & Mean* & $95 \% \mathrm{Cl}$ & Mean* & $95 \% \mathrm{Cl}$ \\
\hline \multicolumn{7}{|c|}{ Eating takeaway foods at the age of 12 years } \\
\hline Less than once per week ( $n$ 448) & 23.0 & 0.3 & $75 \cdot 8$ & 0.8 & 20.5 & 0.6 \\
\hline Once or more per week (n 245) & $23 \cdot 6$ & $1 \cdot 3$ & $75 \cdot 2$ & $3 \cdot 3$ & $21 \cdot 3$ & 2.5 \\
\hline$P$ & \multicolumn{2}{|c|}{0.65} & \multicolumn{2}{|c|}{0.85} & \multicolumn{2}{|c|}{0.72} \\
\hline
\end{tabular}

${ }^{*}$ Adjusted for age, sex, ethnicity, parental education, parental employment, physical activity and energy intake.

differences observed could be because adolescent girls are more likely to under-report food intake, whereas adolescent boys often over-report food intake ${ }^{(27)}$. Overall, our data suggest that public health messages that target adolescents might be needed to help reverse the perception that consuming large portions of takeaway foods is desirable ${ }^{(28)}$.

We also observed frequent consumption of takeaway foods to be associated with reduced consumption of healthy foods such as fruits and vegetables during adolescence, which concurs with other childhood studies ${ }^{(3,4,7)}$. This is an important observation, as it provides further evidence that consumption of unhealthy foods could displace healthy food choices, that is, compromises diet quality during adolescence and could possibly lead to not meeting recommended intakes of key nutrients and food groups ${ }^{(4,7)}$.

Frequency of eating takeaway foods was not associated with adiposity and BP measures. This finding is consistent with a study of 11-14-year-old schoolchildren, which showed that takeaway food consumption was not positively associated with BMI, corrected for age and sex ${ }^{(3)}$. A US survey also observed a non-significant relationship between frequency of eating fast food and being overweight in 11-18-year-old children ${ }^{(29)}$. This could be because schoolchildren under-report the amount of times they were consuming takeaway foods, particularly if they were already overweight or obese ${ }^{(3,23)}$. Second, there is the possibility that our results are influenced by a degree of reverse causation, where those schoolchildren who were already overweight/obese are avoiding takeaway foods in order to reduce their weight ${ }^{(23)}$. Third, it has been suggested that the context in which fast food is consumed may be an important factor in assessing the association between fast food consumption and adiposity ${ }^{(30)}$. For example, it has been shown that television viewing is associated with increased total energy intake in children ${ }^{(31)}$. Therefore, if takeaway food consumption at home occurs in front of the TV rather than away from home, it could show a more marked association with childhood $\operatorname{adiposity}^{(30)}$, and potentially BP. However, in our study, we were not able to disentangle the context in which takeaway foods were consumed, and thus cannot disregard residual confounding from these unmeasured factors influencing observed associations with takeaway foods.

Strengths of this study include its random cluster sample of a large number of representative schoolchildren, prospective design and satisfactory response rate. Further, the validity of the long FFQ tool has been previously reported ${ }^{(13)}$. However, our study is not without its limitations. While we assessed a range of correlates that could influence consumption of takeaway foods during adolescence, we did not have data on societal factors (e.g. peer-group influences) and parental lifestyle and dietary data, which could have confounded the observed associations. In addition, the consumption of takeaway foods in this study was based on a single question; there were no questions to determine the exact kind of takeaway food consumed, and hence we cannot assess the energy density or nutritional content of the food consumed. We also have no indication on the portion size or whether takeaway foods were accompanied by other items such as sugar-sweetened beverages ${ }^{(23)}$. Moreover, validity analysis showed the summation of takeaway-type foods reported in the long FFQ (120-item) provide a higher estimate of takeaway food use compared with the short takeaway food questions ${ }^{(2)}$. However, both methods of comparison rely on longer-term recall of foods consumed, with neither method recording food as consumed via record, which would be preferred for a comparative validity exercise. In addition, the FFQ items from the long FFQ may not necessarily represent takeaway foods, as this was not specifically asked, so some of the food items may have been prepared at home. Therefore, it could be quite reasonable to expect differences between these two recall methods. Although, it does seem likely that those who report consuming $1+$ serves of takeaway foods per week did actually consume this amount while some of the people who report less, may actually have had more than once per week. This would be consistent with other validity studies of short questions about takeaway food use among adolescents $^{(32)}$. Finally, we cannot disregard the possibility of selection bias given that participants who had complete FFQ data compared with those who did not complete the FFQ differed in several baseline characteristics (e.g. sex, ethnicity and systolic BP); hence, this could have influenced observed estimates in our study.

\section{Conclusions}

In summary, we show that a relatively high proportion of schoolchildren consumed takeaway foods at least weekly during adolescence. Frequent takeaway food consumption was not significantly associated with BP or with adiposity measures during adolescence. Only sex was a significant predictor of takeaway food consumption during adolescence. It is likely that other factors such as peer-group influences might play a more important role in consumption of takeaway foods as children become older. Schoolchildren who ate takeaway foods frequently were more likely to make other unhealthy lifestyle choices such as reducing their consumption of fruits and vegetables. 


\section{Acknowledgements}

The Sydney Childhood Eye Study was supported by the Australian National Health and Medical Research Council (253732); the Westmead Millennium Institute, University of Sydney; the Vision Co-operative Research Centre, University of New South Wales, Sydney, and Dairy Australia, Melbourne, Australia. These funding agencies had no influence on the conclusion drawn.

The authors' responsibilities were as follows - B. G. and P. M.: study concept and design; P. M.: acquisition of data; G. B.: analysis of data; B. G., V. M. F., J. C. Y. L., L. A. B. and P. M.: interpretation of data; B. G.: drafting of the manuscript; B. G., V. M. F., G. B., J. C. Y. L., L. A. B. and P. M.: critical revision of the manuscript.

The authors declare that there are no conflicts of interest.

\section{References}

1. Nielsen SJ, Siega-Riz AM \& Popkin BM (2002) Trends in food locations and sources among adolescents and young adults. Prev Med 35, 107-113.

2. Smith KJ, McNaughton SA, Gall SL, et al. (2009) Takeaway food consumption and its associations with diet quality and abdominal obesity: a cross-sectional study of young adults. Int J Behav Nutr Phys Act 6, 29.

3. Patterson R, Risby A \& Chan MY (2012) Consumption of takeaway and fast food in a deprived inner London Borough: are they associated with childhood obesity? BMJ Open 2, e000402.

4. Fraser LK, Edwards KL, Cade JE, et al. (2011) Fast food, other food choices and body mass index in teenagers in the United Kingdom (ALSPAC): a structural equation modelling approach. Int J Obes (Lond) 35, 1325-1330.

5. Astrup A (2005) Super-sized and diabetic by frequent fast-food consumption? Lancet 365, 4-5.

6. Harnack LJ, French SA, Oakes JM, et al. (2008) Effects of calorie labeling and value size pricing on fast food meal choices: results from an experimental trial. Int J Behav Nutr Phys Act 5, 63

7. Bowman SA, Gortmaker SL, Ebbeling CB, et al. (2004) Effects of fast-food consumption on energy intake and diet quality among children in a national household survey. Pediatrics 113, 112-118.

8. Paeratakul S, Ferdinand DP, Champagne CM, et al. (2003) Fast-food consumption among US adults and children: dietary and nutrient intake profile. J Am Diet Assoc 103, 1332-1338.

9. Pereira MA, Kartashov AI, Ebbeling CB, et al. (2005) Fast-food habits, weight gain, and insulin resistance (the CARDIA study): 15-year prospective analysis. Lancet 365, 36-42.

10. Seow DY, Haaland B \& Jafar TH (2015) The association of prehypertension with meals eaten away from home in young adults in Singapore. Am J Hypertens 28, 1197-1200.

11. Adams J, Goffe L, Brown T, et al. (2015) Frequency and sociodemographic correlates of eating meals out and take-away meals at home: cross-sectional analysis of the UK national diet and nutrition survey, waves 1-4 (2008-12). Int J Behav Nutr Phys Act 12, 51.

12. Ojaimi E, Rose KA, Smith W, et al. (2005) Methods for a population-based study of myopia and other eye conditions in school children: the Sydney Myopia Study. Ophthalmic Epidemiol 12, 59-69.
13. Watson JF, Collins CE, Sibbritt DW, et al. (2009) Reproducibility and comparative validity of a food frequency questionnaire for Australian children and adolescents. Int J Behav Nutr Phys Act 6, 62 .

14. Food Standards Australia New Zealand $(2007,2011)$ NUTTAB 2006. Canberra: FSANZ.

15. Food Standards Australia New Zealand (2010) NUTTAB 2010. Canberra: FSANZ

16. Food Standards Australia New Zealand (2007) AUSNUT 2007. Canberra: FSANZ.

17. Bezerra IN, Curioni C \& Sichieri R (2012) Association between eating out of home and body weight. Nutr Rev 70, 65-79.

18. Cole TJ, Bellizzi MC, Flegal KM, et al. (2000) Establishing a standard definition for child overweight and obesity worldwide: international survey. BMJ 320, 1240-1243.

19. Pickering TG, Hall JE, Appel LJ, et al. (2005) Recommendations for blood pressure measurement in humans and experimental animals: part 1: blood pressure measurement in humans: a statement for professionals from the Subcommittee of Professional and Public Education of the American Heart Association Council on High Blood Pressure Research. Hypertension 45, 142-161.

20. Gopinath B, Baur LA, Wang JJ, et al. (2010) Blood pressure is associated with retinal vessel signs in preadolescent children. J Hypertens 28, 1406-1412.

21. Gopinath B, Baur LA, Burlutsky G, et al. (2012) Socio-economic, familial and perinatal factors associated with obesity in Sydney schoolchildren. J Paediatr Child Health 48, 44-51.

22. Gopinath B, Hardy LL, Baur LA, et al. (2012) Physical activity and sedentary behaviors and health-related quality of life in adolescents. Pediatrics 130, e167-e174.

23. Braithwaite I, Stewart AW, Hancox RJ, et al. (2014) Fast-food consumption and body mass index in children and adolescents: an international cross-sectional study. BMJ Open 4, e005813.

24. Baird DL, Syrette J, Hendrie GA, et al. (2012) Dairy food intake of Australian children and adolescents 2-16 years of age: 2007 Australian National Children's Nutrition and Physical Activity Survey. Public Health Nutr 15, 2060-2073.

25. Gopinath B, Flood VM, Burlutsky G, et al. (2014) Pattern and predictors of dairy consumption during adolescence. Asia Pac J Clin Nutr 23, 612-618.

26. Gopinath B, Louie JC, Flood VM, et al. (2014) Influence of obesogenic behaviors on health-related quality of life in adolescents. Asia Pac J Clin Nutr 23, 121-127.

27. Gopinath B, Flood VM, Burlutsky G, et al. (2014) Dairy food consumption, blood pressure and retinal microcirculation in adolescents. Nutr Metab Cardiovasc Dis 24, 1221-1227.

28. Denney-Wilson E, Crawford D, Dobbins T, et al. (2009) Influences on consumption of soft drinks and fast foods in adolescents. Asia Pac J Clin Nutr 18, 447-452.

29. French SA, Story M, Neumark-Sztainer D, et al. (2001) Fast food restaurant use among adolescents: associations with nutrient intake, food choices and behavioral and psychosocial variables. Int J Obes Relat Metab Disord 25, 1823-1833.

30. MacFarlane A, Cleland V, Crawford D, et al. (2009) Longitudinal examination of the family food environment and weight status among children. Int J Pediatr Obes 4, 343-352.

31. Wiecha JL, Peterson KE, Ludwig DS, et al. (2006) When children eat what they watch: impact of television viewing on dietary intake in youth. Arch Pediatr Adolesc Med 160, 436-442.

32. Gwynn JD, Flood VM, D'Este CA, et al. (2010) The reliability and validity of a short FFQ among Australian Aboriginal and Torres Strait Islander and non-Indigenous rural children. Public Health Nutr 14, 388-401. 